\title{
Invited commentary to letter by Jan Lambrecht
}

\author{
P. K. Amid
}

Received: 12 March 2011 / Accepted: 1 April 2011 / Published online: 27 April 2011

(C) Springer-Verlag 2011

Dear Editors,

I read with interest the letter of Dr. Lambrecht in this issue of Hernia. The proposed formula, which is a mathematic expression for establishing an ideal mesh/defect ratio, does not consider the location of the defect or the different methods of ventral hernia repair. It might be useful for open "Onlay", and laparoscopic "IPOM", bearing in mind that the former requires a higher mesh/defect ratio, and the latter is for defects less than $10 \mathrm{~cm}$.

The formula cannot be applied to the open "Retromuscular" repair, which is the most popular and widely accepted open repair. In the Rives/Stoppa retromuscular repair, regardless of defect size, the transverse width of the mesh is the distance between the right and left semilunaris lines, to which the sides of the mesh are to be anchored. The distance between these lines differs in different patients. Also, it does not apply to the vertical length of the defect because, for upper abdominal hernias approaching the xiphoid, the upper limit for mesh extension is the apex of the retro xiphoid triangle, and for lower abdominal defects approaching the pubic bone the lower limit of mesh extension is the Cooper's ligament, to which the lower edge of the mesh is to be anchored.

The same limitations are true for hernias on either side of the midline.

In summary, the proposed coefficient cannot be applied to all ventral hernias as a universal formula.
P. K. Amid ( $\square)$

David Geffen School of Medicine at UCLA,

Lichtenstein Hernia Institute at UCLA, Los Angeles, CA, USA

e-mail: pamid@onemain.com 